\title{
Completion of meiosis in male zebrafish (Danio rerio) despite lack of DNA mismatch repair gene mlh1
}

\author{
Marcelo C. Leal • Harma Feitsma • Edwin Cuppen • \\ Luiz R. França $\cdot$ Rüdiger W. Schulz
}

Received: 3 August 2007 / Accepted: 31 October 2007 / Published online: 5 February 2008

(C) The Author(s) 2007

\begin{abstract}
Mlh1 is a member of DNA mismatch repair (MMR) machinery and is also essential for the stabilization of crossovers during the first meiotic division. Recently, we have shown that zebrafish mlh1 mutant males are completely infertile because of a block in metaphase I, whereas females are fertile but have aneuploid progeny. When studying fertility in males in a two-fold more inbred background, we have however observed low numbers of fertilized eggs (approximately $0.4 \%$ ). Histological examination of the testis has revealed that all spermatogenic stages prior to spermatids (spermatogonia, primary sperma-
\end{abstract}

Marcelo C. Leal and Harma Feitsma contributed equally to this work.

This work was supported by the Brazilian Foundation CAPES, the Cancer Genomics Center (Nationaal Regie Orgaan Genomics), the European Union-funded FP6 Integrated Project ZF-MODELS, and Utrecht University.

M. C. Leal $\cdot$ R. W. Schulz $(\bowtie)$

Science Faculty, Department Biology,

Section Endocrinology \& Metabolism, Utrecht University,

Kruyt Building, Room Z-203, Padualaan 8,

NL-3584 CH Utrecht, The Netherlands

e-mail: r.w.schulz@uu.nl

H. Feitsma $\cdot$ E. Cuppen

Hubrecht Institute for Developmental Biology and Stem

Cell Research, Section Functional Genomics and Bioinformatics,

Uppsalalaan 8,

3584 CT Utrecht, The Netherlands

M. C. Leal • L. R. França

Laboratory of Cellular Biology, Department of Morphology,

Federal University of Minas Gerais,

Minas Gerais, Brazil

R. W. Schulz

Institute of Marine Research,

P.O. Box 1870, Nordnes,

5817 Bergen, Norway tocytes, and secondary spermatocytes) are significantly increased in the mutant, whereas the total weight of spermatids and spermatozoa is highly decreased $(1.8 \mathrm{mg}$ in wild-type vs. $0.1 \mathrm{mg}$ in mutants), a result clearly different from our previous study in which outbred males lack secondary spermatocytes or postmeiotic cells. Thus, a delay of both meiotic divisions occurs rather than complete arrest during meiosis I in these males. Eggs fertilized with mutant sperm develop as malformed embryos and are aneuploid making this male phenotype much more similar to that previously described in the mutant females. Therefore, crossovers are still essential for proper meiosis, but meiotic cell divisions can progress without it, suggesting that this mutant is a suitable model for studying the cellular mechanisms of completing meiosis without crossover stabilization.

Keywords DNA repair enzyme Mlh1 · Testis . Spermatogenesis $\cdot$ Male fertility $\cdot$ Aneuploidy . Zebrafish, Danio rerio (Teleostei)

\section{Introduction}

Mismatch repair machinery corrects errors in newly synthesized DNA. This system is composed of many members, Mlh1 being one of the key proteins (Jun et al. 2006; Jiricny 2006a, b). Several mismatch repair genes also play a role in meiosis (Wei et al. 2002), and the Mlh1 protein is believed to stabilize crossover sites, which are essential for keeping the homologous chromosomes in a paired conformation during the first meiotic division. In prophase of meiosis I, a number of double-strand breaks are generated that are potential recombination sites. Only a fraction of these (one or two per chromosome pair) will be 
repaired by crossing over (Marcon and Moens 2005). During pachytene, Mlh1 forms distinct foci on the synapsed chromosomes; these foci coincide with the sites of crossover in timing, number and position (Marcon and Moens 2003).

Both female and male mlh1 knockout mice are sterile (Baker et al. 1996; Edelmann et al. 1996; Woods et al. 1999; Eaker et al. 2002). However, the examination of gonad histology has revealed a sex difference: males lack spermatozoa completely, whereas females have oocytes, but these are rarely able to finish meiosis (Edelmann et al. 1996; Woods et al. 1999). Synapsis proceeds normally in these mice, but the frequency of crossovers is dramatically reduced, and chromosomes are mostly present as univalents instead of bivalents in metaphase I (Baker et al. 1996; Woods et al. 1999), thus impairing subsequent cell division.

Zebrafish mlh1 knockouts show sex-dependent meiotic problems similar to those of mice. Male mutants are sterile, but females are fertile, giving aneuploid or triploid progeny. In males, spermatogenesis is blocked at metaphase I, because most chromosomes are in a univalent state. This is associated with an accumulation of primary spermatocytes, whereas secondary spermatocytes, postmeiotic spermatids, and spermatozoa are absent. In females, meiotic divisions apparently can progress, although the unpaired chromosomes cause severe mis-segregation. However, during the first cell divisions after fertilization, this does not cause problems, as the zygotic aberrant genomic content is rescued by maternally provided mRNAs and proteins (Feitsma et al. 2007).

We have now crossed the original mlh1 mutant founder back to wild-type fish of its own line twice and show here, for the first time in vertebrates, that homozygous mutant males on this background are able to finish meiosis, albeit with strongly reduced numbers of spermatozoa that also result in aneuploid embryos. This phenotype highly resembles that of female fish but has never been observed in mice. Thus, Mlh1 is still essential for proper meiosis, but cell divisions can progress without functional Mlh1.

\section{Materials and methods}

\section{Zebrafish lines and Western blot}

To obtain mlh1 mutant zebrafish on a more homogeneous background, the founder fish with the original point mutation in mlhl (hu1919) was crossed out twice to wildtype fish of the TL line from which the founder was generated. Two independent in-crosses of heterozygous progeny were then performed, from which homozygous mutant males were used for this study. Genotyping was performed as described previously (Feitsma et al. 2007).
The albino females used for crosses originated from the $\mathrm{b} 4$ line, a line carrying a 4-kb insertion in exon 6 of the zebrafish SLC45A2 gene, which is located on chromosome 21.

Western blot was run on protein extracts of testes of wild-type, heterozygous, and homozygous mutant males. Testis tissue was homogenized in $10 \mathrm{mM}$ TRIS buffer, $\mathrm{pH}$ 7.4, containing $1 \%$ SDS and $1 \mathrm{mM}$ orthovanadate. Proteins were separated by $8 \%$ SDS-polyacrylamide gel electrophoresis. For MLH1 detection, a mouse monoclonal antihuman MLH1 antibody (BD Biosciences Pharmingen, Franklin Lake, N.J.) was used. Tubulin, used as a loading control, was detected by a mouse monoclonal antibody against acetylated alpha-tubulin (Sigma-Aldrich, St. Louis, Mo.). Mouse antibodies were visualized with anti-mouse antibody conjugated to horseradish peroxidase and an ECLdetection kit (Amersham Biosciences, Freiburg, Germany).

Biometry, histology, and morphometry

For biometrical analysis, six wild-type and six mutant males from in-cross 1 together with 12 wild-type and 11 mutant males from in-cross 2 were anesthetized, weighed, and killed. Testes were dissected out, fixed in $4 \%$ glutaraldehyde, weighed, and embedded in 2-hydroxyethyl methacrylate. Sections ( $4 \mu \mathrm{m}$ thick) were stained with toluidine blue and used for histological and morphometric evaluation.

For morphometric analysis of testis tissue from the inbred TL line (12 wild-type and 11 mutant males from incross 2), the weights of the various testicular tissue components were determined by using a 441-point intersection grid placed in the ocular of a light microscope. Fifteen fields chosen randomly (6,615 points) were scored for each animal at a magnification of $\times 400$. Staining or sectioning artifacts were rarely seen and were not considered in the total number of points utilized to obtain weights. Intersection points were classified as being over one of the following components: spermatogonia, primary spermatocytes, secondary spermatocytes, spermatids/spermatozoa, apoptotic cells, or others (somatic cells, blood and lymphatic vessels, connective tissue, and empty spaces). The scores were first expressed as the volume fraction (\%) per testis tissue component. The weight (mg) of each testis component was then determined as the product of the volume fraction (\%) and the total testis weight, as described in Feitsma et al. (2007).

All data are presented as the mean \pm SEM and analyzed via analysis of variance (Student-Newman-Keuls test). The significance level in comparisons was considered to be $P<0.05$.

TUNEL method

Detection of apoptotic cells was performed in 5- $\mu \mathrm{m}$-thick Bouin-fixed paraffin-embedded testis sections $(n=3$ per 
genotype) that were incubated for $5 \mathrm{~min}$ in $10 \mathrm{mM}$ citric buffer ( $\mathrm{pH} 6.0$ ) at $98^{\circ} \mathrm{C}$ and then allowed to cool down to room temperature. Endogenous peroxidase was blocked with $3 \% \mathrm{H}_{2} \mathrm{O}_{2}$ in MilliQ (MQ) for $5 \mathrm{~min}$. Sections were washed three times with phosphate-buffered saline (PBS) before incubation for $1 \mathrm{~h}$ in 5-triphosphate-biotin nick-end labeling (TUNEL) mix at $37^{\circ} \mathrm{C}$. The TUNEL mix consisted of $0.3 \mathrm{U} / \mu \mathrm{l}$ calf thymus terminal deoxynucleotidyl transferase (Amersham Biosciences), $6.66 \mu \mathrm{M} / \mu \mathrm{l}$ biotin dUTP (Roche, Basel, Switzerland) in terminal transferase buffer (Amersham Biosciences). The reaction was stopped by incubating the sections in $300 \mathrm{mM} \mathrm{NaCl}, 30 \mathrm{mM}$ sodium citrate in MQ for $15 \mathrm{~min}$ at room temperature. After washes with PBS, non-specific staining was blocked by incubation with $2 \%$ bovine serum albumin (BSA; Sigma) in PBS at room temperature for $10 \mathrm{~min}$, before the sections were treated for $30 \mathrm{~min}$ at $37^{\circ} \mathrm{C}$ in a moist chamber with a $1: 20$ dilution of ExtrAvidin peroxidase antibody. After three washes in PBS, detection was performed with diaminobenzidine (DAB+; DAKO, Glostrup, Denmark). Sections were counterstained with Mayer's hematoxylin, dehydrated, and mounted with Pertex (Cellpath, Hemel Hempstead, UK).

\section{Histone H3 immunodetection}

Proliferation of spermatogonia and primary spermatocytes was assessed by phosphorylated histone $\mathrm{H} 3 \mathrm{(pH} 3$ ) immunodetection. Histone $\mathrm{H} 3$ is a chromosomal protein component involved in the condensation of mitotic and meiotic chromosomes and becomes phosphorylated during late G2 phase, being present until metaphase in the cell cycle (Hendzel et al. 1997; Cobb et al. 1999), i.e., it is detectable in cells preparing to divide. Testes were fixed in Bouin ( $n=6$ per genotype), embedded in paraffin, and sectioned. The sections were mounted on glass slides coated with 3-aminopropyl triethoxysilane (Sigma), dried overnight at $37^{\circ} \mathrm{C}$, deparaffinized, and rehydrated before incubation in a plastic chamber filled with $1 \mathrm{mM}$ EDTA solution containing $0.05 \%$ Tween 20, pH 8.0 (Merck-Schuchardt, Hohenbrunn, Germany). For epitope retrieval, the chamber was transferred to a boiling-water bath for $20 \mathrm{~min}$ and then left to cool to room temperature (RT). Nonspecific protein-binding sites were blocked with $5 \%$ goat serum (Vector Laboratories, Burlingame, Calif.)plus 1\% BSA (Sigma) in PBS for $30 \mathrm{~min}$, followed by incubation with a polyclonal rabbit anti-human phospho-histone $\mathrm{H} 3$ IgG preparation (Upstate, Charlottesville, USA; 1:200 dilution in $1 \%$ BSA in PBS) for $1 \mathrm{~h}$ at RT. After being rinsed in PBS, sections were immersed in $0.35 \%$ hydrogen peroxide in PBS for $10 \mathrm{~min}$ to quench endogenous peroxidase activity. The subsequent incubation with biotinylated goat anti-rabbit $\operatorname{IgG}(1: 100$, Vector Laboratories) in $1 \%$ BSA in PBS lasted $30 \mathrm{~min}$ at RT, after which the slides were incubated with $\mathrm{ABC}$ (avidin-biotin complex; Vector Laboratories) for $1 \mathrm{~h}$, according to the manufacturer's protocol. DAB (DAKO) substrate development was peformed for $30 \mathrm{~s}$. Nuclei were counterstained and mounted as described above. For negative controls, the primary antibody was replaced by the same concentration of normal rabbit IgG (Santa Cruz Biotechnology, Santa Cruz, Calif., USA).

Sequencing of single nucleotide polymorphisms and chromosome spreads

Embryonic DNA was amplified and resequenced by using specific forward (5'-AGTGAAGGGCTTCATCTCC-3') and reverse (5'-AAGTAGTGCATCTATTGAAAATG-3') primers for the polymorphism in the mlhl gene (located on chromosome 13). Peak area proportions were determined visually. Chromosome spreads were performed as described previously (Feitsma et al. 2007).

\section{Results}

Male mutant phenotype

Thirty eight homozygous mutant males were used for repeated crosses. To our surprise, they turned out to be not completely infertile, although only very few eggs became fertilized. In total, from 118 crosses, 28 contained fertilized eggs, with 86 out of 22,266 eggs having been fertilized (average fertilization $=0.4 \%$ ). Histological analysis of the testes revealed that post-meiosis II stages of spermatogenesis, spermatids, and spermatozoa were readily observable in wild-type testes in the seminiferous tubules (Fig. 1a), with abundant spermatozoa in the efferent ducts (Fig. 1a, inset). In mutant testes, however, the weight of post-meiotic cells was strongly reduced (Fig. 1b,c). Nevertheless, and in accordance with the (limited) fertility described above, some spermatozoa were visible in the efferent ducts of all mutant animals (Fig. 1b, inset). This showed that some germ cells were able to complete both meiotic divisions and spermiogenesis in the mutant testis. Further, in the mutant testes, secondary spermatocytes were released into the lumen of the seminiferous tubules, indicating that precocious release of germ cells from the germinal epithelium had occurred (Fig. 1b). A large number of apoptotic figures was observed in the mutants.

The body weight was similar in wild-type and mutant males (data not shown). However, the testis weight was significantly higher in mutants $(5.3$ vs. $4.0 \mathrm{mg} ; P<0.05)$, also resulting in a significantly $(P<0.05)$ higher gonadosomatic index (GSI: the ratio between the testes weight and body weight), being $1.3 \%$ and $1.1 \%$, in mutant and wildtype males, respectively. 


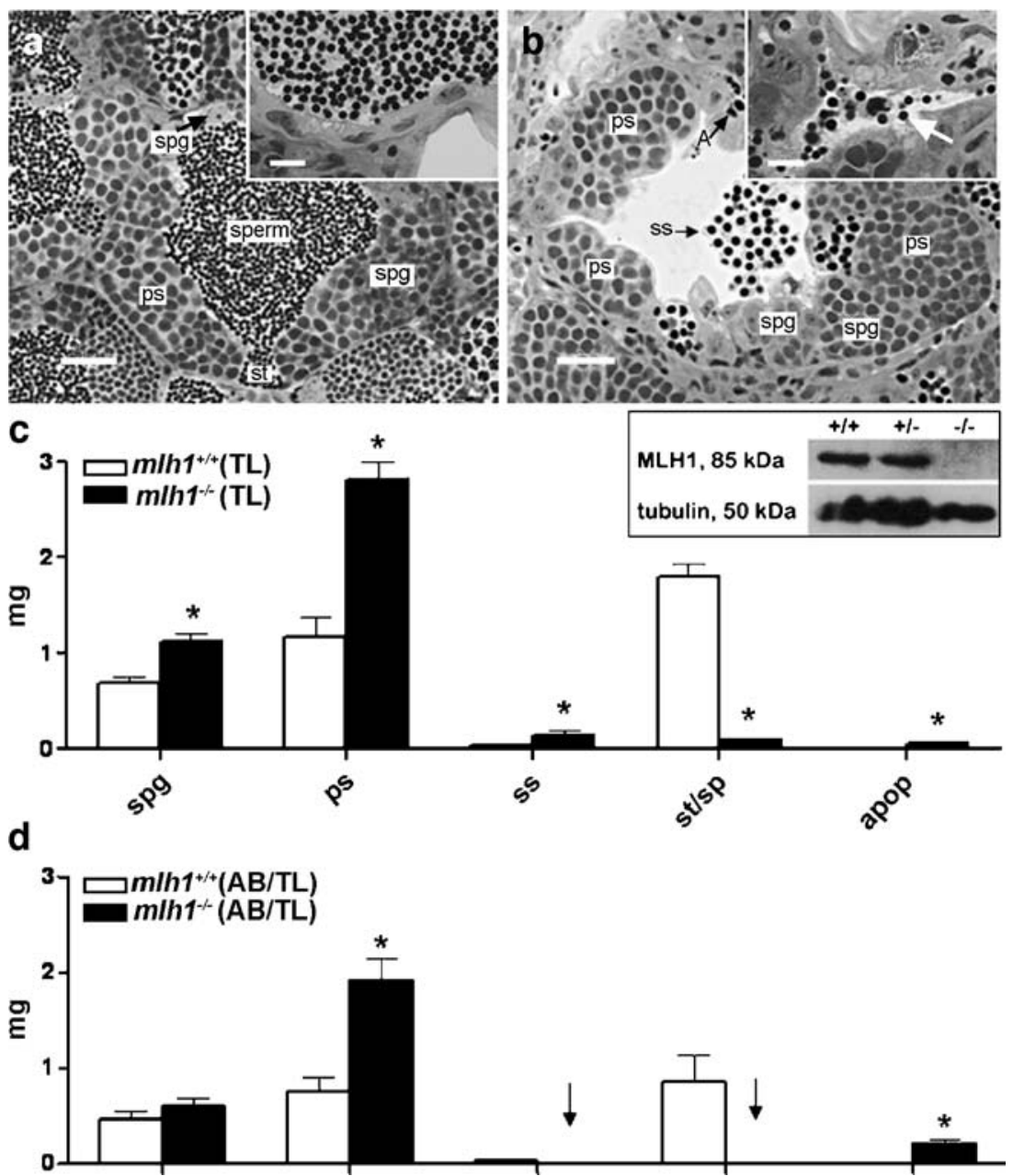

Fig. 1 Testis histology of $m l h 1^{-/}$zebrafish. a, b Cross section of seminiferous tubule in $m l h 1^{+/+}$and $m l h 1^{-/-}$zebrafish, respectively, showing spermatogenic cysts with various types of germ cells: different types of spermatogonia ( $s p g)$, primary spermatocytes $(p s)$, secondary spermatocytes $(s s)$, spermatids $(s t)$, spermatozoa (sperm), and apoptotic cells $(A)$. Note the abnormal spermatogenesis in the mutant with many secondary spermatocytes having been released into the lumen, and very few sperm being produced (b, inset, arrow), whereas numerous spermatozoa are visible in the tubular lumen and efferent ducts of wild-type males (a, inset). Bars $25 \mu \mathrm{m}(\mathbf{a}, \mathbf{b}), 10 \mu \mathrm{m}$ (insets) c Morphometric analysis of testis sections from fish with TL background. The mutant shows significantly higher amounts of spermatogonia (spg), primary spermatocytes $(p s)$, secondary sperma-

Quantitative morphometrical analysis of testis tissue components showed that the mass of spermatogonia and spermatocytes (mainly primary but also secondary) was significantly higher in mutant than in wild-type testes (Fig. 1b,c). We also recorded an increase in the mass of apoptotic cells, but the absolute mass changes were small compared with those recorded for spermatogonia and spermatocytes. In addition, tissue components summarized as "others" (somatic cells, blood and lymphatic vessels, connective tissue, and empty space) were higher $(P<0.05)$ in the mutants compared with those of wild-type fish (1.13 vs $0.34 \mathrm{mg}$, respectively), mainly representing the empty space tocytes ( $s$ ), and apoptotic cells (apop) than the wild-type. On the other hand, spermatids and spermatozoa $(s t / s p)$ are significantly reduced. Inset: Western blot of testes proteins stained with antibodies for MLH1 (85 kDa) and tubulin (50 kDa). MLH1 protein is completely absent in $m l h 1^{-/}$testes $(-/-)$but detectable in wild-type $(+/+)$ and heterozygous $(+/-)$ males. d Morphometric analysis of testis sections from wild-type and $m l h 1^{-/}$mutant zebrafish on an $\mathrm{AB} /$ TL background ( $n=6$ for both genotypes). The mutant shows an increased mass of spermatogonia (not significant), primary spermatocytes, and apoptotic cells. Secondary spermatocytes, spermatids, and spermatozoa were completely absent (arrows). Significant differences between wild-type and mutant genotypes are indicated $(* P<0.05)$

in the lumen of the seminiferous tubules occupied by spermatozoa in wild-type males (Fig. 1a,b). Hence, spermatids/ spermatozoa were almost absent $(0.1 \mathrm{mg}$ or $\sim 1.7 \%$ of the testis parenchyma) compared with wild-type animals (1.8 $\mathrm{mg}$ or $\sim 45 \%$ of the testis parenchyma; Fig. 1a-c). The weight increase of spermatogonia and spermatocytes slightly exceeded the loss of post-meiotic cells in the mutant; this, together with the probably fluid-filled empty space, explained the overall weight gain of mutant testes. Finally, in mutant testis, groups of cells with strongly condensed nuclei could be observed, viz., cells in apoptosis (Fig. 1b), whereas wild-type testis showed low levels of apoptosis in 
Fig. 2 Testis immunohistochemistry of $\mathrm{mlh}^{-/}$zebrafish. a, b TUNEL staining of $m l h 1^{+/+}$ and $m l h 1^{-/}$fish, respectively. Large numbers of apoptotic cells can be seen in mutant testis (brown). Wild-type testis exhibits a low incidence of apoptosis. c, $\mathbf{d}$ Histone $\mathrm{H} 3$ staining of cells in metaphase from $m l h 1^{+/+}$and $m l h 1^{-/}$fish, respectively. Note the higher incidence of stained cells in mutant testis indicating a higher number of mitotic and meiotic cells in metaphase. Bars $25 \mu \mathrm{m}(\mathbf{a}, \mathbf{b}), 50 \mu \mathrm{m}(\mathbf{c}, \mathbf{d})$
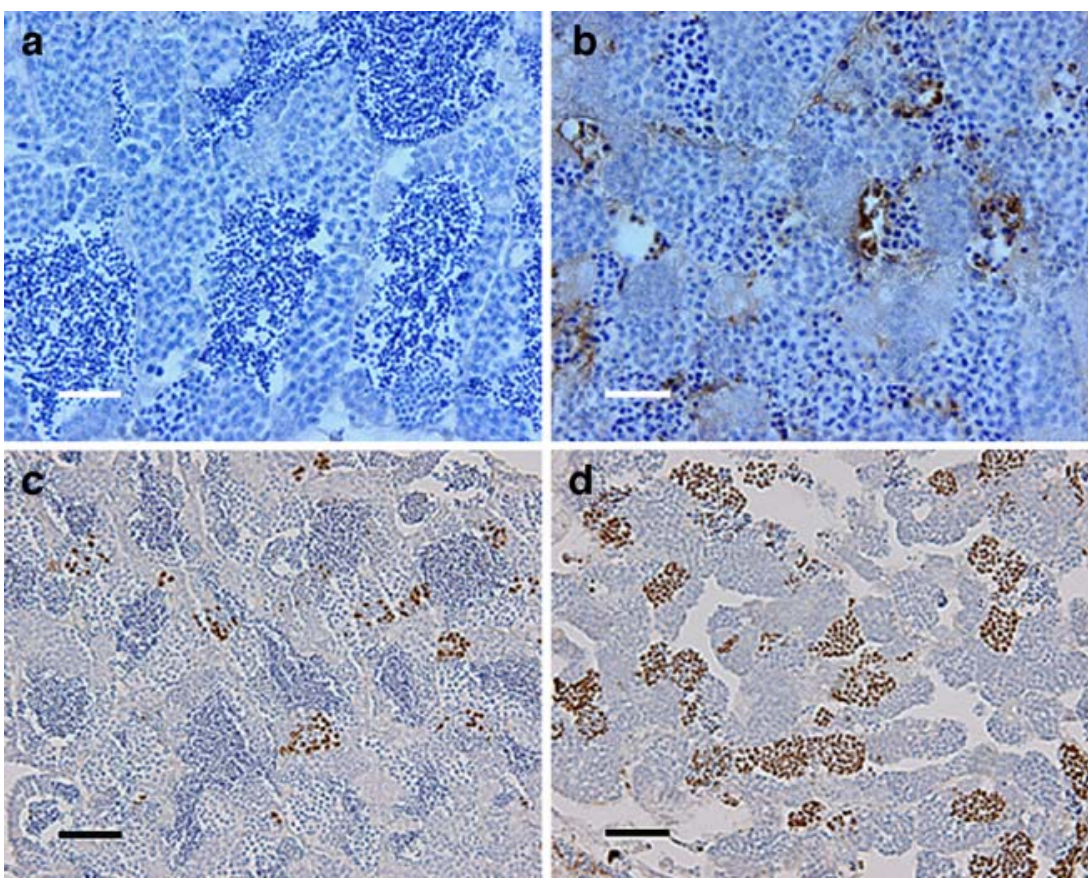

sections stained with toluidine blue (Fig. 1a). The elevated incidence of apoptosis (mainly primary and secondary spermatocytes) in mutant testis and the low number of apoptotic cells in wild-type males was confirmed by TUNEL-based staining of DNA fragments (Fig. 2a,b).

A comparison of the morphometric data of the inbred TL line (Fig. 1c) with data from completely infertile animals carrying the same mutation on a different genetic background $\left(\mathrm{AB} / \mathrm{TL} ; n=6\right.$ for wild-type and for $m l h 1^{-/-}$ genotype; Feitsma et al. 2007) revealed a major difference as regards secondary spermatocytes and postmeiotic germ cells (Fig. 1d). These more advanced stages were not found in $\mathrm{AB} / \mathrm{TL}$ outbred mutant males. In the TL inbred mutant males, on the contrary, the weight of secondary spermatocytes increased above wild-type levels, and postmeiotic cells were found in all samples, albeit at greatly reduced levels compared with wild-type animals.

Histone $\mathrm{H} 3$ staining of testis sections indicated a clear increase in the number of immunopositive cells in mutant compared with wild-type testis (Fig. 2c,d), demonstrating an increase of the proportion of both spermatogonia and spermatocytes in the metaphase stage of the cell cycle.

\section{Progeny}

Most of the fertilized eggs developed into abnormal embryos that died early during development (Fig. 3a), suggesting that they were aneuploid because of meiotic mis-segregation similar to the progeny from $m l h 1$ mutant females. Additionally, mutant males were crossed with females that were homozygous mutant for the albino gene, located on chromosome 21 . This would normally result in albino heterozygous and thus wild-type pigmented progeny, but in this case, some unpigmented progeny were observed (Fig. 3b), indicative of the loss of the wild-type paternal copy of the albino gene by some form of genomic instability. Sequencing the mlhl DNA polymorphism on chromosome 13 in 24-h-old progeny of mutant males showed that four out of ten cases had no or two copies of the paternal chromosome 13 (Fig. 3c). Finally, chromosome numbers of similar progeny deviated from the normal number of 50 (Fig. 3d), with a distribution of chromosome numbers around 50 and close to 75 (Fig. 3e) similar to the distribution in progeny from mutant females (Feitsma et al. 2007), although no completely triploid embryos were found in the current set. Taken together, this indicated that the embryos from eggs fertilized by mlh1 mutant males were aneuploid, and that the meiotic mis-segregation resembled that in mutant females.

\section{Discussion}

Previously, we have reported that male zebrafish lacking the Mlh1 protein are infertile, because spermatogenesis arrests at the metaphase of meiosis I, and the cells die by apoptosis, so that later germ cell stages are absent (Feitsma et al. 2007). This has been explained by the absence of chiasmata, which normally hold homologous chromosomes together. In $m l h 1^{-/}$mutants, the chromosomes of the primary spermatocytes are therefore present as univalents, which impairs chromosome alignment, spindle formation, 

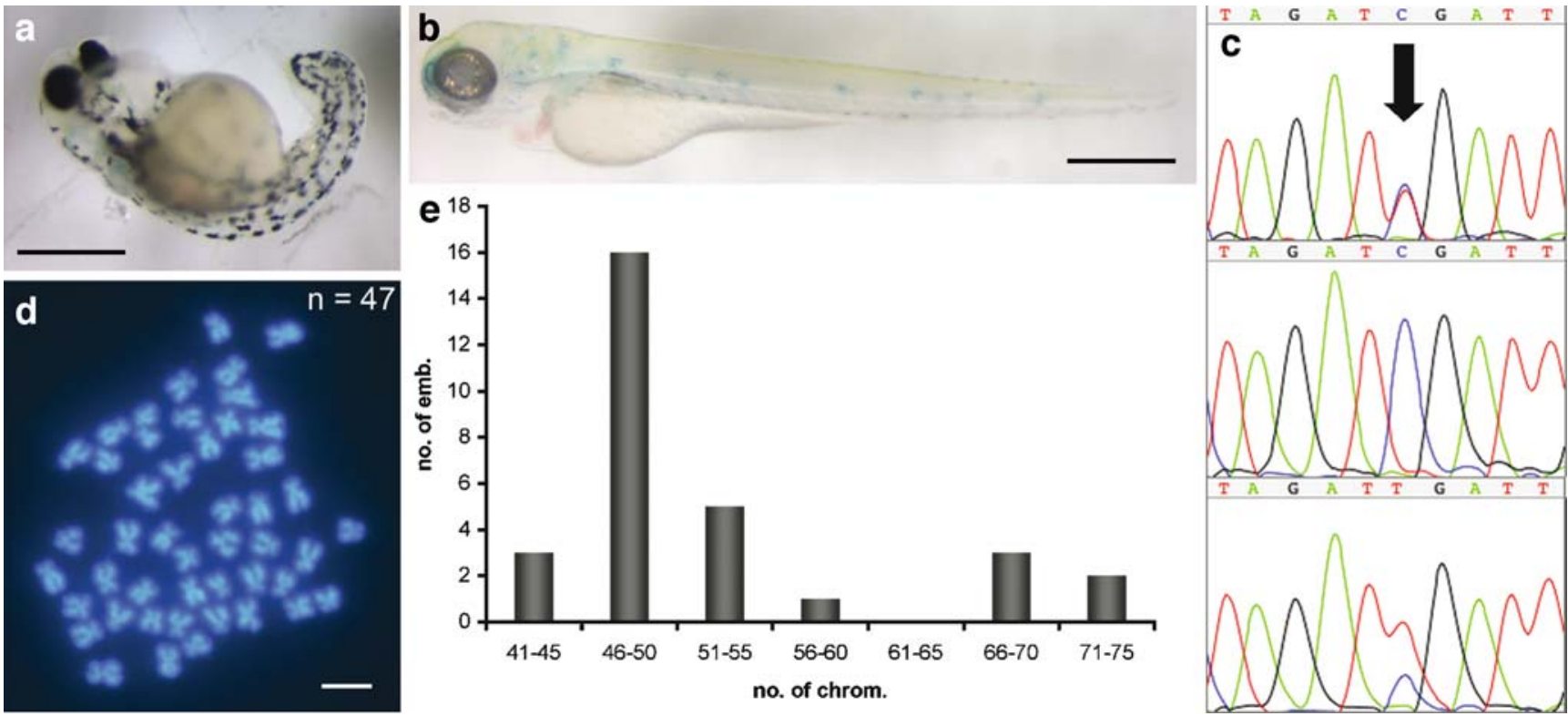

Fig. 3 Progeny of $m l h 1^{-/}$males. a Severely malformed 3-day-old embryo from a cross of a $m l h 1^{-/}$male with a wild-type female. b Unpigmented 3-day-old embryo from a cross of a $m l h 1^{-1-}$ male with an albino female, showing that the wild-type paternal allele of the albino gene has been lost in this embryo. The weak blue staining is caused by the methylene-blue-containing medium. c Sequence traces of the polymorphism in $m l h 1$ (arrow). The paternal allele (mutant) is a $\mathrm{T}$, and the maternal allele (wild-type) is a $\mathrm{C}$. The sequence peak areas

and thus cell division. In contrast, in $m l h 1^{-/-}$females, a lack of chiasmata does not block cell division but causes random chromosome segregation resulting in aneuploid and triploid progeny. Sex differences have also been seen in several mouse knockouts for meiotic genes (Hunt and Hassold 2002). These sex differences in outcomes of meiotic problems are related to general differences in male and female meiosis. In mammals, male gametogenesis is a continuous, tightly regulated process. In contrast, females have a single programmed wave of gametogenesis with a limited number of oocytes. In fish, however, oogonia are present in the adult ovary (Higashino et al. 2002; Grier et al. 2007) allowing for the high female fertility typical of many teleost species.

Here, we show that $m l h 1^{-/}$zebrafish males that are twofold more inbred than those previously studied are partially fertile. Males of the inbred TL line are different from the outbred $(\mathrm{AB} / \mathrm{TL})$ males, which carry the same mutation but are completely infertile with a general arrest in metaphase I (Feitsma et al. 2007), as they do not show a complete block at metaphase I but rather an accumulation of all pre-meiotic and meiotic stages and of apoptotic cells. As a consequence, the inbred line exhibits low levels of post-meiotic cells, associated with low fertilization rates. The observation of secondary spermatocyte weight being higher than in wild-type testis indicates that these cells are not escapees of a meiosis I checkpoint, but that, instead, both meiotic divisions are delayed. The meiotic phenotype show quantitatively that some progeny of mutant males have one chromosomal copy of each parent (top), whereas others have only the maternal allele (middle) or two copies of the paternal allele (bottom). d Embryos have chromosome numbers different from the normal number of 50, such as this example of 47 chromosomes. e Distribution of chromosome numbers in embryos (emb.) from $m l h 1^{-/-}$males (30 embryos in total). Bars $500 \mu \mathrm{m}(\mathbf{a}, \mathbf{b}), 1 \mu \mathrm{m}(\mathbf{d})$

of these males is therefore markedly different from that of the outbred males from our previous study. Additionally, we have shown that the low number of mutant germ cells that complete meiosis and spermiogenesis are aneuploid spermatozoa, because they result in aneuploid progeny upon fertilization of wild-type eggs. This has been demonstrated in three different ways: (1) fertilization of eggs of an albino female sometimes results in unpigmented embryos because of the loss of the paternal allele, (2) sequencing of the $m l h 1$ polymorphism in progeny shows the loss or duplication of the paternal allele, (3) counts of chromosomes of embryonic cells reveals numbers differing from 50 . Together, these data indicate that, in the absence of Mlh1, spermatogenesis still can be completed by a limited number of germ cells but is certainly abnormal. The problems during the first meiotic division are probably attributable to the presence of univalent chromosomes as previously described (Feitsma et al. 2007), whereas the problems during meiosis II are possibly related to the cells being aneuploid, even at this stage. This is consistent with our observation of high numbers of histone-H3-positive primary and secondary spermatocytes in the mutant testis indicating that most cells are delayed at metaphase.

The $m l h 1^{-/-}$females of these crosses behave similarly to the outbred females in terms of fertilization and numbers of phenotypically abnormal embryos (results not shown). In the specific line described here, female and male responses 
to meiotic problems are therefore highly comparable and different from higher vertebrates. The determination of such low fertilization may be experimentally only feasible in fish models, such as zebrafish and medaka (Oryzias latipes) as a result of the high numbers of eggs produced. However, to our knowledge, no spermatozoa or other post-meiosis I stages have ever been seen in mouse mlhl knockout males, and oocytes are also hardly able to finish meiosis (Edelmann et al. 1996; Woods et al. 1999; Lipkin et al. 2002). This suggests that, specifically in zebrafish (or perhaps more generally in lower vertebrates), meiotic divisions are less restricted. As we have shown previously, the germ cells of triploid male zebrafish are also able to complete meiosis (Feitsma et al. 2007).

The difference in male fertility between the two fish lines that we have used is so far unexplained. One possible explanation is that the lines have a genetic modifier background that, for example, decreases or increases the restrictiveness of meiotic checkpoints. Alternatively, sequence divergence between homologous chromosomes might result in a smaller capacity to finish meiosis. In yeast, high levels of polymorphisms have been shown to decrease the frequency of recombination (Borts and Haber 1987). Together with a severe reduction in recombination because of the absence of Mlh1, such a decrease might pass a certain threshold below which meiosis I is completely blocked. In this respect, a cross between this $m l h 1$ mutant with mutants for mismatch repair genes that are not essential for crossover formation, but that are involved in the repair of heteroduplex sequences that arise during meiotic recombination, such as $m s h 2$ and msh6 (Borts et al. 2000; Radford et al. 2007), might be of interest. Experimental evidence for each explanation would need long-term experiments involving in- and outcrosses with different lines. This would certainly be feasible in zebrafish but is beyond the scope of this study. Whichever explanation turns out to be true, both sexes of this mutant line seem suitable models for investigations into the cellular mechanisms allowing meiosis to be completed in the absence of Mlh1 protein in some of the mutant germ cells.

Open Acces This article is distributed under the terms of the Creative Commons Attribution Noncommercial License which permits any noncommercial use, distribution, and reproduction in any medium, provided the original author(s) and source are credited.

\section{References}

Baker SM, Plug AW, Prolla TA, Bronner CE, Harris AC, Yao X, Christie DM, Monell C, Arnheim N, Bradley A, Ashley T, Liskay
RM (1996) Involvement of mouse Mlh1 in DNA mismatch repair and meiotic crossing over. Nat Genet 13:336-342

Borts RH, Haber JE (1987) Meiotic recombination in yeast: alteration by multiple heterozygosities. Science 237:1459-1465

Borts RH, Chambers SR, Abdullah MF (2000) The many faces of mismatch repair in meiosis. Mutat Res 451:129-150

Cobb J, Miyaike M, Kikuchi A, Handel MA (1999) Meiotic events at the centromeric heterochromatin: histone $\mathrm{H} 3$ phosphorylation, topoisomerase II alpha localization and chromosome condensation. Chromosoma 108:412-425

Eaker SJ, Cobb J, Pyle A, Handel MA (2002) Meiotic prophase abnormalities and metaphase cell death in MLH1-deficient mouse spermatocytes: insights into regulation of spermatogenic progress. Dev Biol 249:85-95

Edelmann W, Cohen PE, Kane M, Lau K, Morrow B, Bennett S, Umar A, Kunkel T, Cattoretti G, Chaganti R, Pollard JW, Kolodner RD, Kucherlapati R (1996) Meiotic pachytene arrest in MLH1-deficient mice. Cell 85:1125-1134

Feitsma H, Leal MC, Moens PB, Cuppen E, Schulz RW (2007) Mlh1 deficiency in zebrafish results in male sterility and aneuploid as well as triploid progeny in females. Genetics 175: $1561-1569$

Grier HJ, Uribe MC, Parenti LR (2007) Germinal epithelium, folliculogenesis, and postovulatory follicles in ovaries of rainbow trout, Oncorhynchus mykiss (Walbaum, 1792) (Teleostei, Protacanthopterygii, Salmoniformes). J Morphol 268:293-310

Hendzel MJ, Wei Y, Mancini MA, Van Hooser A, Ranalli T, Brinkley BR, Bazett-Jones DP, Allis CD (1997) Mitosis-specific phosphorylation of histone $\mathrm{H} 3$ initiates primarily within pericentromeric heterochromatin during G2 and spreads in an ordered fashion coincident with mitotic chromosome condensation. Chromosoma 106:348-360

Higashino T, Miura T, Miura C, Yamauchi K (2002) Histological studies on early oogenesis in barfin flounder (Verasper moseri). Zool Sci 19:557-563

Hunt PA, Hassold TJ (2002) Sex matters in meiosis. Science 296:2181-2183

Jiricny J (2006a) The multifaceted mismatch-repair system. Nat Rev Mol Cell Biol 7:335-346

Jiricny J (2006b) MutL $\alpha$ : at the cutting edge of mismatch repair. Cell 126:239-241

Jun SH, Kin TG, Ban C (2006) DNA mismatch repair system: classical and fresh roles. FEBS J 273:1609-1619

Lipkin SM, Moens PB, Wang V, Lenzi M, Shanmugarajah D, Gilgeous A, Thomas J, Cheng J, Touchman JW, Green ED, Schwartzberg P, Collins FS, Cohen PE (2002)Meiotic arrest and aneuploidy in MLH3-deficient mice. Nat Genet 31:385-390

Marcon E, Moens P (2003) MLH1p and MLH3p localize to precociously induced chiasmata of okadaic-acid-treated mouse spermatocytes. Genetics 165:2283-2287

Marcon E, Moens PB (2005) The evolution of meiosis: recruitment and modification of somatic DNA-repair proteins. Bioessays 27:795-808

Radford SJ, Sabourin MM, McMahan S, Sekelsky J (2007) Meiotic recombination in Drosophila Msh6 mutants yields discontinuous gene conversion tracts. Genetics 176:53-62

Wei K, Kucherlapati R, Edelmann W (2002) Mouse models for human DNA mismatch-repair gene defects. Trends Mol Med 8:346-353

Woods LM, Hodges CA, Baart E, Baker SM, Liskay M, Hunt PA (1999) Chromosomal influence on meiotic spindle assembly: abnormal meiosis I in female Mlh1 mutant mice. J Cell Biol 145:1395-1406 